\title{
Monthly reservoir inflow forecasting using a new hybrid SARIMA genetic programming approach
}

\author{
Hamid Moeeni, Hossein Bonakdari* and Isa Ebtehaj \\ Department of Civil Engineering, Razi University, Kermanshah, Iran. \\ ${ }^{*}$ Corresponding author. e-mail: bonakdari@yahoo.com
}

Forecasting reservoir inflow is one of the most important components of water resources and hydroelectric systems operation management. Seasonal autoregressive integrated moving average (SARIMA) models have been frequently used for predicting river flow. SARIMA models are linear and do not consider the random component of statistical data. To overcome this shortcoming, monthly inflow is predicted in this study based on a combination of seasonal autoregressive integrated moving average (SARIMA) and gene expression programming (GEP) models, which is a new hybrid method (SARIMA-GEP). To this end, a four-step process is employed. First, the monthly inflow datasets are pre-processed. Second, the datasets are modelled linearly with SARIMA and in the third stage, the non-linearity of residual series caused by linear modelling is evaluated. After confirming the non-linearity, the residuals are modelled in the fourth step using a gene expression programming (GEP) method. The proposed hybrid model is employed to predict the monthly inflow to the Jamishan Dam in west Iran. Thirty years' worth of site measurements of monthly reservoir dam inflow with extreme seasonal variations are used. The results of this hybrid model (SARIMA-GEP) are compared with SARIMA, GEP, artificial neural network $(\mathrm{ANN})$ and SARIMA-ANN models. The results indicate that the SARIMA-GEP model $\left(R^{2}=78.8\right.$, $\mathrm{VAF}=78.8, \mathrm{RMSE}=0.89, \mathrm{MAPE}=43.4, \mathrm{CRM}=0.053)$ outperforms SARIMA and GEP and SARIMAANN $\left(R^{2}=68.3, \mathrm{VAF}=66.4, \mathrm{RMSE}=1.12, \mathrm{MAPE}=56.6, \mathrm{CRM}=0.032\right)$ displays better performance than the SARIMA and ANN models. A comparison of the two hybrid models indicates the superiority of SARIMA-GEP over the SARIMA-ANN model.

\section{Introduction}

Time series analysis has an important role in the hydrologic sciences and water resource management. Time series modelling of river flow discharge is of particular importance in water resource management. Time series models are used to forecast the future based on past observations (Makridakis et al. 2008). Statistical modelling is the oldest method of time series prediction. Seasonal Autoregressive Integrated Moving Average (SARIMA) is considered the best statistical linear type of model in runoff prediction (Valipour 2015) and has been widely employed in studies on flow (Mohan and Vedula 1995; Chebaane et al. 1995; Wang et al. 2005; Yurekli and Kurunc 2005; Yurekli et al. 2005; Abebe and Foerch 2008; Aksoy et al. 2013; Ali 2013; Valipour 2015). Since SARIMA performs linear modelling, it does not perform well with complex nonlinear problems.

Soft computing techniques perform well with complex nonlinear problems, so these approaches have been well-received in recent decades (Kilinç and Cigizoglu 2005; Kisi and Cigizoglu 2007; Firat 2008; Lohani et al. 2012; Ebtehaj and Bonakdari 2013; He et al. 2014; Kothari and Gharde 2015;

Keywords. Forecasting; inflow; time series; hybrid; gene expression programming. 
Sharma et al. 2015; Khoshbin et al. 2016). Unlike traditional statistical methods, soft computing functions are based on artificial intelligence. Moreover, time series prediction models have different structures and functions. Therefore, recognising the strengths and weaknesses of each method is very important. Among soft computing methods, artificial neural network (ANN) modelling is used extensively. A number of researchers have studied the performance of ANN modelling against statistical modelling in discharge flow prediction. Kisi (2004) and Mohammadi et al. (2005) compared ANN models with Autoregressive (AR) and Autoregressive Integrated Moving Average (ARIMA) models in predicting discharge. The results indicated that ANN modelling is more accurate. Valipour et al. (2013) studied reservoir inflow and found that ANN models are better than Autoregressive Moving Average (ARMA) and ARIMA models. Another artificial intelligence method is Gene Expression Programming (GEP), which performs based on the theory of natural evolution and has high prediction ability. Numerous studies have been done in various fields to compare ANN performance. Some researchers have studied the performance of stochastic models against ANN models in predicting hydrological series. Wang et al. (2009) compared the performance of different models in predicting monthly river inflow and found that genetic programming (GP) outperforms ANN and SARIMA models. Kisi et al. (2012a) took into account the daily discharge as input to several models and predicted the daily sediment load. They concluded that GEP modelling is more powerful than ANN. Kisi et al. (2012b) predicted daily lake levels using artificial intelligence models and found that, compared with other models such as ANN, GEP has greater ability.

Both soft computing and statistical modelling methods have certain limitations in predicting time series. Thus, some researchers have attempted to use the advantages of both methods, simultaneously, to overcome the disadvantages of each method in the proposed hybrid method. Zhang (2003) presented a hybrid ARIMA-ANN model and modelled the residual of ARIMA with ANN. This model is based on the assumption that the data have linear and nonlinear components. The results demonstrated that using the hybrid ARIMA-ANN increases modelling accuracy, as the results of both ARIMA and ANN are better. ARIMA-ANN hybrid model performance has been evaluated in other studies as well (Koutroumanidis et al. 2009; Faruk 2010; Nourani et al. 2011; Liu et al. 2012; Filho et al. 2014). Moeeni and Bonakdari (2016) enhanced the accuracy of monthly discharge forecasting with irregular seasonal fluctuations using SARIMA-ANN modelling.
According to recent studies, compared to ANN, GEP shows greater power in nonlinear system modelling. Therefore, a new hybrid of SARIMA and GEP is presented (SARIMA-GEP) in this research to overcome the problems of existing hybrid methods in estimating time series. To the best of the authors' knowledge, this method has not been used in predicting time series with severe and irregular seasonal fluctuations. The monthly inflow of the Jamishan River located in the west of Iran is predicted with the hybrid SARIMAGEP model. To evaluate the performance of the proposed model, the results are compared with a SARIMA statistical model, two computational intelligence-based models (ANN and GEP), and one hybrid method that is presented in this study, i.e., SARIMA-ANN.

\section{Materials and methods}

\subsection{Proposed hybrid process}

In general, the modelling process consists of four steps: (1) data pre-processing, (2) linear modelling by SARIMA, (3) nonlinear evaluation of the SARIMA model residuals, and (4) nonlinear modelling of residuals by GEP. Figure 1 illustrates these four steps for a variable such as monthly inflow $Q(t)$ based on the hybrid SARIMA-GEP model. Monthly inflow $Q(t)$ comprises deterministic $(D(t))$ and stochastic $(S(t))$ components as follows:

$$
Q(t)=D(t)+S(t)
$$

It is better to delete the deterministic component in the data pre-processing step before modelling and only model the stochastic component (Salas et al. 1988; Marco et al. 2012). This stochastic term can have a linear $\left(S_{L}(t)\right)$ or nonlinear $\left(S_{N}(t)\right)$ structure, meaning it can be modelled with linear and nonlinear models, respectively. But if the term includes both of these components (equation 2), the structure cannot be modelled properly.

$$
S(t)=S_{L}(t)+S_{N}(t) .
$$

To solve this problem, a hybrid model is presented, in which $S_{L}(t)$ is modelled with a linear model and $S_{N}(t)$ with a nonlinear model. The linear model is used initially and then its residual is assumed to be a nonlinear component $\left(S_{N}(t)\right)$. This assumption is correct when the residual series based on the respective test has no deterministic and linear terms. Then this series is modelled with a nonlinear model, and a nonlinear component $\left(\hat{S}_{N}(t)\right)$ is thus obtained. If the sum of the linear component $\left(\hat{S}_{L}(t)\right)$ and deterministic component modelled (which were removed in the 


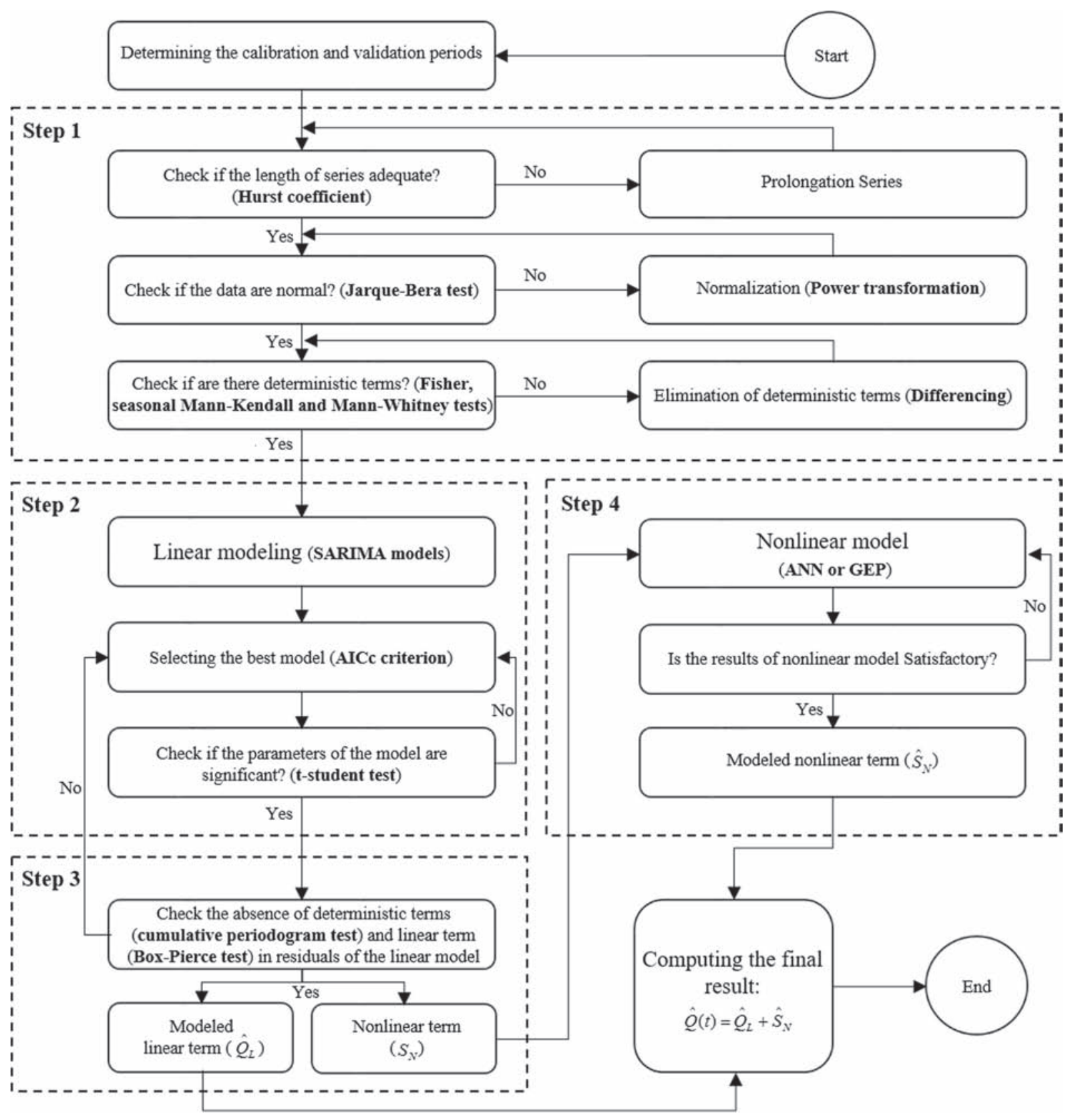

Figure 1. Proposed hybrid process flowchart.

pre-processing step) are denoted by $\hat{Q}_{L}(t)$, the end result will be

$$
\hat{Q}(t)=\hat{Q}_{L}(t)+\hat{S}_{N}(t) .
$$

Figure 1 shows the proposed four-step hybrid process. The steps include data pre-processing, linear modelling, nonlinear term verification and nonlinear modelling, as discussed below.

\subsubsection{Series pre-processing}

A series study should include all short- and longterm series of wet and dry periods. This criterion is evaluated using the Hurst coefficient $(H)$. If the value is $>0.5$, the series is sufficiently long. The Hurst coefficient is given as follows:

$$
H=\frac{\log \left(S_{\max }-S_{\min } / S_{d}\right)}{\log (N / 2)} .
$$

Here $S_{\min }$ and $S_{\max }$ are the minimum and maximum cumulative differences from the mean, $S_{d}$ is the standard deviation and $N$ is the number of months.

A substantial assumption in many statistical models is that the series is normal. The power 
transformation for data normalisation and the Jarque-Bera normality test are used as follows:

$$
\begin{gathered}
Q_{n}(t)=\left\{\begin{array}{ll}
\left((Q(t)+\alpha)^{\lambda}-1\right) / \lambda, & \lambda \neq 0 \\
\log (Q(t)+\alpha), & \lambda=0
\end{array} .\right. \\
J_{B}=N\left(\frac{S_{k}^{2}}{6}+\frac{\left(K_{u}-3\right)^{2}}{24}\right) .
\end{gathered}
$$

Here $Q(t)$ and $Q_{n}(t)$ are the original and normalised monthly inflow series, $\alpha$ and $\lambda$ are the power transformation parameters, $S_{k}$ and $K_{u}$ are skewness and kurtosis and $J_{B}$ is the Jarque-Bera test statistic.

The period $(P(t))$, trend $(T(t))$ and jump $(J(t))$ terms are deterministic components (equation 7 ) that should be evaluated before modelling, and if they exist, they should be removed. The components are determined with the Fisher, seasonal Mann-Kendall and Mann-Whitney tests, and are eliminated by non-seasonal and seasonal differencing. After differencing, it is necessary to ensure these components have been removed by doing the relevant tests again.

$$
D(t)=P(t)+T(t)+J(t) .
$$

With the Fisher test, the existence of a periodic term is checked by defining the Fisher test statistic $\left(F^{*}\right)$ as follows (Kashyap and Rao 1976):

$$
F^{*}=\frac{N(N-2)\left(\alpha_{k}^{2}+\beta_{k}^{2}\right)}{4\left(\sum_{k=1}^{N / 2}\left(Q(t)-\alpha_{k} \cos \left(\Omega_{k} t\right)-\beta_{k} \sin \left(\Omega_{k} t\right)\right)\right)},
$$

where $\alpha_{k}$ and $\beta_{k}$ are Fourier series expansion coefficients and $\Omega_{k}$ is the angular frequency.

If periodicity is significantly large, the series has a periodic term.

The periodicity corresponding to $\Omega_{k}$ is significant only if

$$
F^{*} \geq F(2, N-2)
$$

where $F$ denotes the critical value in the F-distribution, which is 3.00 at the $5 \%$ significance level for large degrees of freedom in the denominator.

The non-parametric seasonal Mann-Kendall trend test is (Hirsch and Slack 1984)

$$
\begin{gathered}
M_{K}=\sum_{k=1}^{\omega}\left(s_{k}-\operatorname{sgn}\left(s_{k}\right)\right) . \\
s_{k}=\sum_{i=1}^{n_{k}} \sum_{j=i+1}^{n_{k}-1} \operatorname{sgn}\left(x_{k i}-x_{k j}\right) .
\end{gathered}
$$

$\operatorname{var}\left(M_{K}\right)=\sum_{k=1}^{\omega} \frac{n_{k}\left(n_{k}-1\right)\left(2 n_{k}+5\right)}{18}+2 \sum_{i=1}^{\omega-1} \sum_{j=i+1}^{\omega} \sigma_{i j}$.

Here $M_{K}$ is the seasonal Mann-Kendall test statistic, $s_{k}$ is the Mann-Kendall statistic for the $k$ th season, $\operatorname{var}\left(M_{K}\right)$ is the variance of $M_{K}, \omega$ is the number of seasons, and $\sigma_{i j}$ is the test statistic covariance in the $i$ th and $j$ th seasons.

If $u_{M_{K}}=M_{K} \operatorname{var}\left(M_{K}\right)^{-0.5}$ is applied to the following equation, the series without the trend term would be at the $5 \%$ significance level:

$$
\left|u_{M_{K}}\right|<1.96 \text {. }
$$

The Mann-Whitney test to evaluate the presence of a jump is (Mann and Whitney 1947):

$$
u_{M W}=\frac{\sum_{t=1}^{n_{1}}\left(R(z(t))-\frac{n_{1}\left(n_{1}+n_{2}+1\right)}{2}\right)}{\left(\frac{n_{1} n_{2}\left(n_{1}+n_{2}+1\right)}{12}\right)^{1 / 2}},
$$

where $z(t)$ is the series $Q(t)$ arranged as ascending, $R(z(t))$ is the degree of the $z(t)$ function, and $n_{1}$ and $n_{2}$ are the numbers of members in the $Q_{1}(t)=\left\{Q(1), Q(2), \ldots, Q\left(n_{1}\right)\right\}$ and $Q_{2}(t)=$ $\left\{Q\left(n_{1}+1\right), Q\left(n_{1}+2\right), \ldots, Q(n)\right\}$ sub-series. If the corresponding probability with $\left|u_{M W}\right|$ in the normal distribution $\left(P_{\left|u_{M W}\right|}\right)$ is greater than the desired confidence level (here 1\%), the assumption of the two mean series' equality is confirmed. As a result, the series is without jump.

\subsubsection{Linear modelling}

Seasonal Autoregressive Integrated Moving Average (SARIMA) is a statistical linear model that is capable of modelling seasonal series and is expressed as follows:

$$
\begin{aligned}
& \operatorname{SARIMA}(\boldsymbol{p}, \boldsymbol{d}, \boldsymbol{q})(\boldsymbol{P}, \boldsymbol{D}, \boldsymbol{Q})_{!}: \\
& \begin{array}{c}
\left(1-\Phi_{1} B^{\omega}-\Phi_{2} B^{2 \omega}-\cdots-\Phi_{P} B^{P \omega}\right) \\
\quad \times\left(1-\phi_{1} B-\phi_{2} B^{2}-\cdots-\phi_{p} B^{p}\right) \\
\quad \times\left(1-B^{\omega}\right)^{D}(1-B)^{d} Q_{n}(t) \\
=\left(1-\Theta_{1} B^{\omega}-\Theta_{2} B^{2 \omega}-\cdots-\Theta_{Q} B^{Q \omega}\right) \\
\quad \times\left(1-\theta_{1} B-\theta_{2} B^{2}-\cdots-\theta_{q} B^{q}\right) e(t)
\end{array}
\end{aligned}
$$

where $p$ and $q$ are the non-seasonal autoregressive and moving average orders, $P$ and $Q$ are the seasonal autoregressive and moving average orders, $\varphi$ and $\theta$ are the non-seasonal autoregressive and moving average parameters, $\Phi$ and $\Theta$ are the seasonal autoregressive and moving average parameters, $\omega$ is the frequency period, $B$ is the differential operator as $B(Q(t))=Q(t-1),\left(1-B^{\omega}\right)^{D}$ is equal to the $D$ th seasonal differencing with $\omega$ steps, and $(1-B)^{d}$ is equal to the $d$ th non-seasonal differencing. 
Regarding the order of any parameter between 0 and $5,(p, q, P, Q \in\{0,1,2,3,4,5\}), 4$ sets with 6 members are used. All possible combinations of these four sets are $6^{4}=1296$. As a result, 1296 SARIMA models were obtained. Among these, the best model was selected based on Akaike's information criterion as follows:

$$
\mathrm{AICc}=n \cdot \operatorname{Ln}\left(\sigma_{e}^{2}\right)+\frac{2 k n}{n-k-1},
$$

where $\sigma_{e}^{2}$ is the variance of the model residuals, $k$ is the number of model parameters as $p+q+P+Q$ and $n$ is the number of modelled data.

Upon selecting the best model, the parameters' significance was determined using the t-student test (equation 17). If the corresponding probability with this test statistic in the normal distribution $\left(P_{\tau}\right)$ is less than the $5 \%$ confidence level, the desired parameter is significant. Otherwise, another model is chosen based on AICc.

$$
\tau=\frac{\hat{P}_{r}}{S_{e}}
$$

where $\tau$ is the $t$-student test statistic, $\hat{P}_{r}$ represents the model parameters and $S_{e}$ is the standard error.

\subsubsection{Nonlinear term verification}

The Box-Pierce test was used for the reliability of a lack of linear dependence and the cumulative periodogram test was used to ensure the absence of periodicity in the linear model residuals.

If the probability associated with the Box-Pierce test statistic $\left(Q^{*}\right)$ in chi-squared distribution $\left(P_{Q^{*}}\right)$ is greater than the significance level considered (here 5\%), the linear model residuals $(e(t))$ are independent. A cumulative periodogram test diagram was made by sampling to show the cumulative periodogram $\left(P_{i}\right)$ against each frequency $\left(h_{j}\right)$. If this graph is close to the exact line of the $(0,0)$ and $(0.5,1)$ points, the period is completely removed from the residuals. $Q^{*}, P_{i}$ and $h_{i}$ are defined as follows (Box and Pierce 1970; Dagum et al. 1975):

$$
\begin{gathered}
Q^{*}=n(n+2) \sum_{l=1}^{L} \frac{r_{l}^{2}(e(t))}{n-1} . \\
P_{i}=\frac{\begin{array}{c}
2 \sum_{j=1}^{i}\left(\sum_{t=1}^{n} e(t) \cos \left(2 \pi h_{j} t\right)\right)^{2} \\
\times\left(\sum_{t=1}^{n} e(t) \sin \left(2 \pi h_{j} t\right)\right)^{2}
\end{array}}{n^{2} \sigma_{e}^{2}}, \\
i=1,2,3, \ldots, \frac{n}{2} . \\
h_{j}=\frac{j}{n} .
\end{gathered}
$$

Here $r_{l}$ and $\sigma_{e}^{2}$ are the autocorrelation coefficients with the $l$ th delay and variance of $e(t)$, and $L$ is considered the maximum time delay.

\subsubsection{Nonlinear modelling}

The last step in the proposed hybrid process is nonlinear modelling, where gene expression programming (GEP) is used.

Genetic programming (GP) is an artificial intelligence approach presented by Koza (1992) to expand on the genetic algorithm (GA). GP uses various computer programs and offers a method of calculating the target parameter. Gene Expression Programming (GEP) is a linear expression of the GP method. In the GEP method, the phenotype and genotype of each individual are clearly distinct, and each individual can be expressed as a linear sequence (Ferreira 2001). The GEP method generally has five different components, including the function set, fitness function, terminal set, control parameters and stop criteria. According to GEP, genetic operators perform at the chromosome level, hence there is great genetic diversity accordingly. Another reason why GEP is a highly capable method is its unique multigenic nature that leads to the evolution of complex programs consisting of several subprograms. Each gene consists of a symbol list with fixed length, which contains function set or terminal set elements. The function set includes mathematical operators (basic or userdefined functions), while the terminal set contains logical and numerical constants.

The chromosomes used in GEP include a set of linear symbolic strings made from one or two genes. A gene has a head and a tail. The head contains signs and represents terminals in addition to functions, while the tail is only made of terminals. Since there is no explicit method to determine the length of the head, this parameter should be determined by trial and error (Ferreira 2006; Alavi and Gandomi 2011; Ebtehaj et al. 2015). After determining the head length, the tail length is calculated as follows:

$$
t=h\left(n_{\max }-1\right)+1
$$

where $t$ and $h$ are the lengths of the tail and head, respectively, and $n_{\max }$ is the maximum number of functions. The chromosome length is equal to the sum of $h$ and $t$.

The Artificial Neural Network (ANN) is another nonlinear model that can be used in the hybrid process. One of the known structures of artificial neural networks is the multilayer perceptron (MLPNN) that uses a multi-input vector parameter, has a nonlinear relationship, and models the target parameter (monthly inflow in this study). The MLPNN structure has input, hidden and 
output layers. There can be several different hidden layers. Each layer has some weighted sets of entries called neurons. Each neuron has a bias component as well. These neurons use a transfer function to transmit the results to the next layer. The output of this network is the discharge predicted for a double-layer structure (a hidden layer and output layer) as follows:

$$
Q_{t+1}=a_{0}+\sum_{j=1}^{g} a_{i} f\left(b_{0 j}+\sum_{i=1}^{h} b_{i j} Q_{t+1-i}\right)+\varepsilon_{t+1}
$$

where $Q_{t+1}$ is the predicted discharge, $a_{i}$ and $b_{i}$ are model parameters (connection weights), $g$ is the number of input nodes, $h$ is the number of hidden nodes, $f$ is the transfer function and $\varepsilon_{t+1}$ is the model error.

In this work, two-layer and three-layer perceptrons were used for modelling, including Sigmoid transfer functions (equation 23) in the hidden layers. The numbers of hidden layer neurons considered were from 1 to 30 . These models were trained with the Levenberg-Marquardt (LM) algorithm (Levenberg 1944) and then the best model was selected based on the error criteria.

$$
f(t)=\frac{1}{1+e^{-t}}
$$

\subsection{Determining the input combinations}

The SARIMA model inputs are determined by this model order: $p, q, P$ and $Q$. The maximum parameter order of the model can be identified from ACF and PACF diagrams (figure 2). As these graphs have large values in the two initial basic steps, a maximum level of 2 is enough for each parameter (Cryer and Chen 2008). However, Valipour et al.'s (2013) results showed that increasing this number may help achieve a more accurate model. The reason for selecting a maximum order of five parameters for the model is the same.

In order to determine the different inputs for nonlinear models (individual and hybrid models) the hydrological nature of monthly inflow was used. It is obvious that the monthly inflow is mainly affected by the discharge of a few months before and the same month in one or a few years ago. This is evident from the ACF diagram as well. Therefore, 14 input types were considered based on the appropriate time delays in accordance with table 1 for each nonlinear model. The time delays shown in table 1 were used for individual models of discharge $(Q)$ and for hybrid models of SARIMA model residuals.

\subsection{Goodness-of-fit of model performance}

In order to evaluate and compare the models, the correlation coefficient $\left(R^{2}\right)$, variance account for (VAF), root mean squared error (RMSE), mean absolute percentage error (MAPE) and coefficient of residual mass (CRM) criteria were used as shown below. By employing these concurrently, a comprehensive model performance result can be achieved:

$$
R^{2}=\frac{\left(\sum_{t=1}^{n}(Q(t)-\bar{Q}(t))(\hat{Q}(t)-\overline{\hat{Q}}(t))\right)^{2}}{\sum_{t=1}^{n}(Q(t)-\bar{Q}(t))^{2} \sum_{t=1}^{n}(\hat{Q}(t)-\overline{\hat{Q}}(t))^{2}} .
$$

Table 1. Input combinations for soft computing models.

\begin{tabular}{ll}
\hline Model ID & \multicolumn{1}{c}{ Time delays } \\
\hline Model 1 & $t-1$ \\
Model 2 & $t-1, t-2$ \\
Model 3 & $t-1, t-12$ \\
Model 4 & $t-1, t-6$ \\
Model 5 & $t-1, t-6, t-12$ \\
Model 6 & $t-1, t-2, t-12$ \\
Model 7 & $t-1, t-2, t-6$ \\
Model 8 & $t-1, t-2, t-3$ \\
Model 9 & $t-1, t-2, t-6, t-12$ \\
Model 10 & $t-1, t-2, t-3, t-12$ \\
Model 11 & $t-1, t-2, t-3, t-6$ \\
Model 12 & $t-1, t-2, t-3, t-6, t-12$ \\
Model 13 & $t-1, t-2, t-3, t-12, t-24$ \\
Model 14 & $t-1, t-2, t-3, t-6, t-12, t-24$ \\
\hline
\end{tabular}

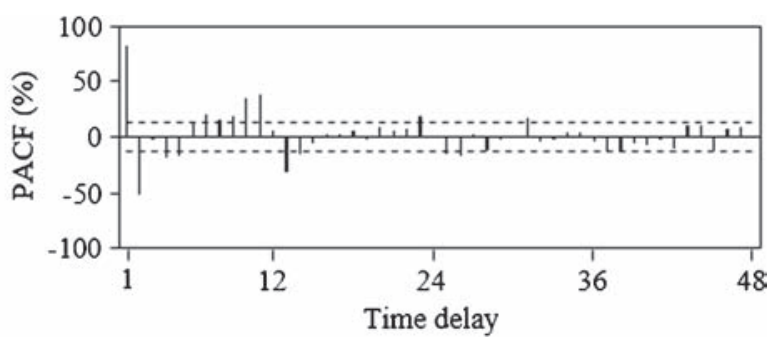

Figure 2. ACF and PACF diagrams. 

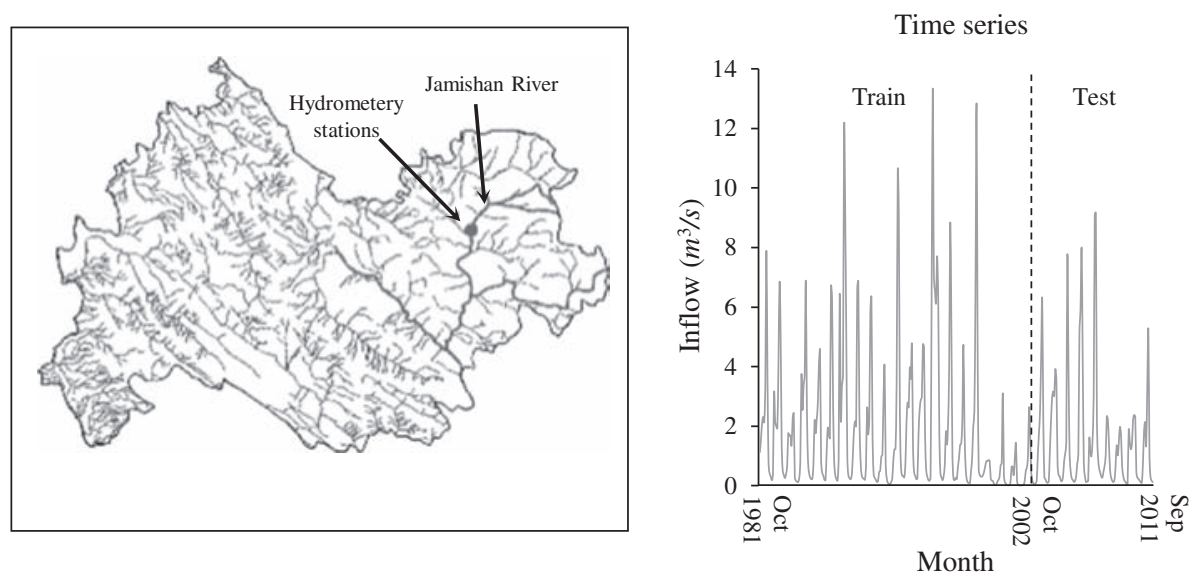

Figure 3. Study area location.

Table 2. Statistical properties of the data used.

\begin{tabular}{lcccccccccc}
\hline Period & $\bar{x}$ & $x_{\min }$ & $x_{\max }$ & $x_{50}$ & $S_{D}$ & $C_{v}$ & $S_{k}$ & $K_{u}$ & $Q_{1}$ & $Q_{3}$ \\
\hline Calibration & 1.9 & 0.01 & 13.3 & 0.8 & 2.4 & 1.30 & 2.2 & 5.2 & 0.3 & 2.4 \\
Prediction & 1.5 & 0.04 & 9.2 & 0.9 & 1.9 & 1.26 & 2.2 & 5.1 & 0.2 & 2.1 \\
\hline
\end{tabular}

$\bar{x}$ : mean, $x_{\min }$ : minimum, $x_{\max }$ : maximum, $x_{50}$ : median, $S_{D}$ : standard deviation, $C_{v}$ : coefficient of variation, $S_{k}$ : skewness, $K_{u}$ : kurtosis, $Q_{1}$ : first quarter; and $Q_{3}$ : third quarter.

Table 3. Test results for the detection of deterministic terms.

\begin{tabular}{llcc}
\hline Test statistics & Series & Result & $\begin{array}{c}\text { Critical } \\
\text { value }\end{array}$ \\
\hline Fisher $\left(F_{\max }^{*}\right)$ & Original & 134.56 & 3.00 \\
& Differenced & -0.17 & 3.00 \\
Seasonal Mann-Kendall $\left(\left|u_{M_{K}}\right|\right)$ & Original & 4.69 & 1.96 \\
& Differenced & 0.03 & 1.96 \\
Mann-Whitney $\left(P_{\left|u_{M W}\right|}\right)$ & Original & 1.29 & 1.00 \\
\hline
\end{tabular}

$F_{\text {max }}^{*}$ : maximum fisher test statistics, $\left|u_{M_{K}}\right|$ : absolute of the corresponding index with seasonal Mann-Kendall test statistic in the standard normal distribution, $P_{\left|u_{M W}\right|}$ : corresponding probability of Mann-Whitney test statistics.

$$
\begin{aligned}
& \mathrm{VAF}=\left(1-\frac{\operatorname{var}(Q(t)-\hat{Q}(t))}{\operatorname{var}(Q(t))}\right) \times 100 . \\
& \mathrm{RMSE}=\sqrt{\frac{\sum_{t=1}^{n}(Q(t)-\hat{Q}(t))^{2}}{n}} . \\
& \mathrm{MAPE}=\frac{\sum_{t=1}^{n}\left|\frac{Q(t)-\hat{Q}(t)}{Q(t)}\right|}{n} \times 100 . \\
& \mathrm{CRM}=\frac{\sum_{t=1}^{n} Q(t)-\sum_{t=1}^{n} \hat{Q}(t)}{\sum_{t=1}^{n} Q(t)} .
\end{aligned}
$$

Table 4. Significance test results for the SARIMA $(1,0,2)$ $(0,1,1)_{12}$ model coefficients.

\begin{tabular}{lcllr}
\hline Parameter & $\varphi_{1}$ & $\theta_{1}$ & $\theta_{2}$ & $\Theta_{1}$ \\
\hline$\hat{P}_{r}$ & 0.9 & 0.195 & 0.149 & 0.92 \\
$T$ & 21.71 & 2.46 & 1.98 & 20.76 \\
$P_{\tau}(\%)$ & 0.0 & 1.4 & 4.8 & 0.0 \\
\hline
\end{tabular}

$\hat{P}_{r}$ : estimated model parameters (such as $\left.\varphi, \theta, \Theta\right), \tau: \mathrm{t}-$ student test statistics, $P_{\tau}$ : corresponding probability of $\tau$ in t-distribution.

\subsection{Study area}

To model and predict the monthly inflow of the Jamishan River in Kermanshah Province, west Iran, data from 1981 to 2011 was used. The reference measurement was done at Pirsalman hydrometric station, where the discharge was measured 
daily and the monthly average was calculated. The station is located at longitude $34^{\circ} 14^{\prime} 17^{\prime \prime}$ and latitude $46^{\circ} 40^{\prime} 12^{\prime \prime}$. Figure 3 shows the study area location. The maximum daily and monthly inflow achieved for the mentioned period is 48 and $13.3 \mathrm{~m}^{3} / \mathrm{sec}$, respectively. This series with strong seasonal fluctuations can be used to evaluate the performance of different models. The first 21 years of data were considered for calibration and the last 9 years' data for prediction. Table 2 shows the statistical properties of the data. The similarity between the statistical properties of calibration

Table 5. Non-linearity test for the linear model residuals.

\begin{tabular}{lclll}
\hline$L$ & 12 & 24 & 36 & 48 \\
$Q^{*}$ & 2.5 & 19.5 & 27.3 & 41.2 \\
$P_{Q^{*}}(\%)$ & 93 & 42.4 & 65.7 & 55 \\
\hline
\end{tabular}

$L$ : considered maximum lag in Box-Pierce test, $Q$ : BoxPierce test statistics and $P_{Q^{*}}$ : corresponding probability of $Q^{*}$ in chi-squared distribution.

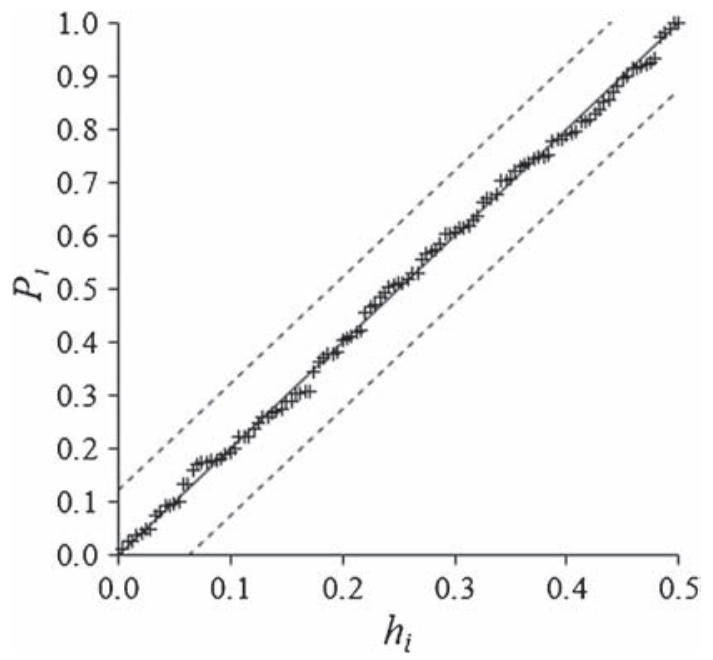

Figure 4. Cumulative periodogram for linear model residuals. and prediction data represents suitable selection of the two periods.

\section{Inflow modelling using a hybrid method}

The Hurst coefficient obtained for the calibration period was 0.71, which represents a sufficient period length for modelling. The value of this coefficient for the entire study series was the same. It means that adding a prediction period to the series will not change the coefficient value. Therefore, it can be concluded that the prediction period length was selected well. The Jarque-Bera test statistics value obtained for the original series was 459.86 . Since the critical value is 5.99 , the series is not normal. After applying power transformation, the test statistic value achieved was 4.21 , which represents a normal series after conversion. Table 3 shows the evaluation test results for the presence of deterministic terms in the series before and after differencing. The results are related to the Fisher, seasonal Mann-Kendall and Mann-Whitney tests, which were used for the periodic term, trend term and jump term, respectively. The Fisher test statistics were calculated for all possible angular frequencies $(N / 2)$, among which, the largest value obtained $\left(F_{\max }^{*}\right)$ was for the original series related to the 12 -month delay frequency $\left(\Omega_{12}\right)$. Since this value is larger than the critical value, the studied series has a periodic term. Thus, one time differencing with a step of 12 months is required. The test statistic value is greater than the critical value at the $5 \%$ confidence level that indicates a trend term in the series. However, the Mann-Whitney test at the confidence level of $1 \%$ indicates the lack of a jump in the series. It can be seen from the table that after differencing all completely deterministic terms have been removed from the series. Hence, series differencing will produce the same stochastic term.
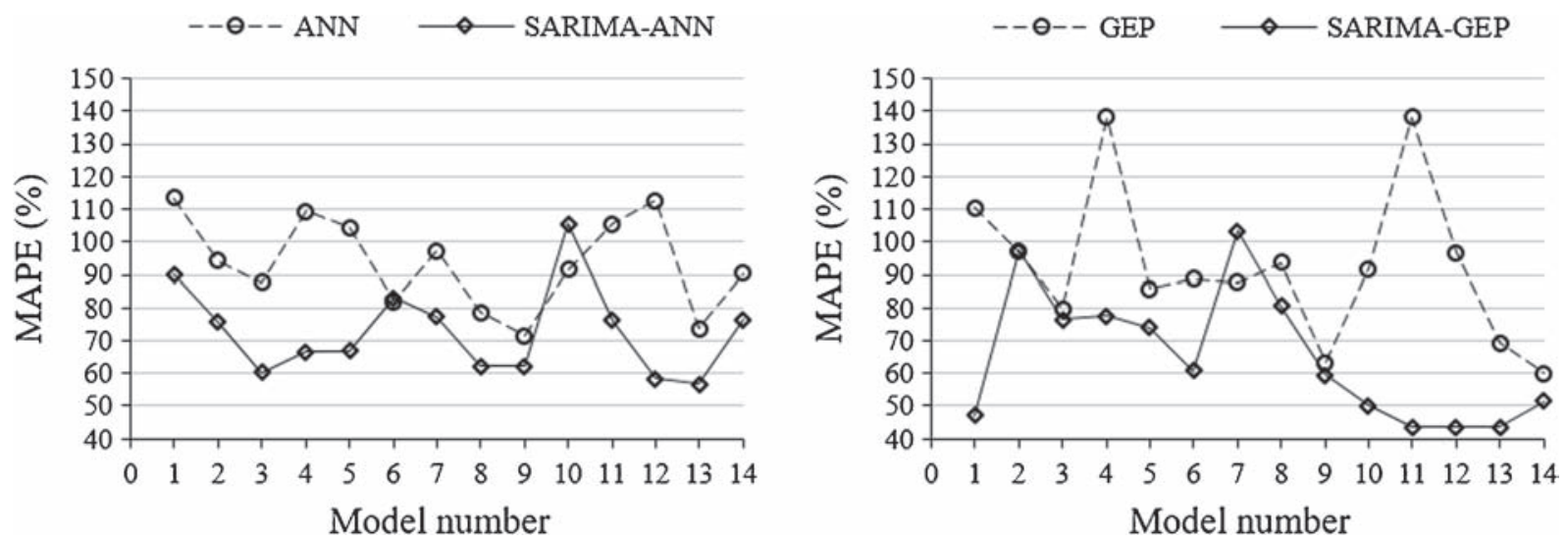

Figure 5. Model results based on different input combinations. 

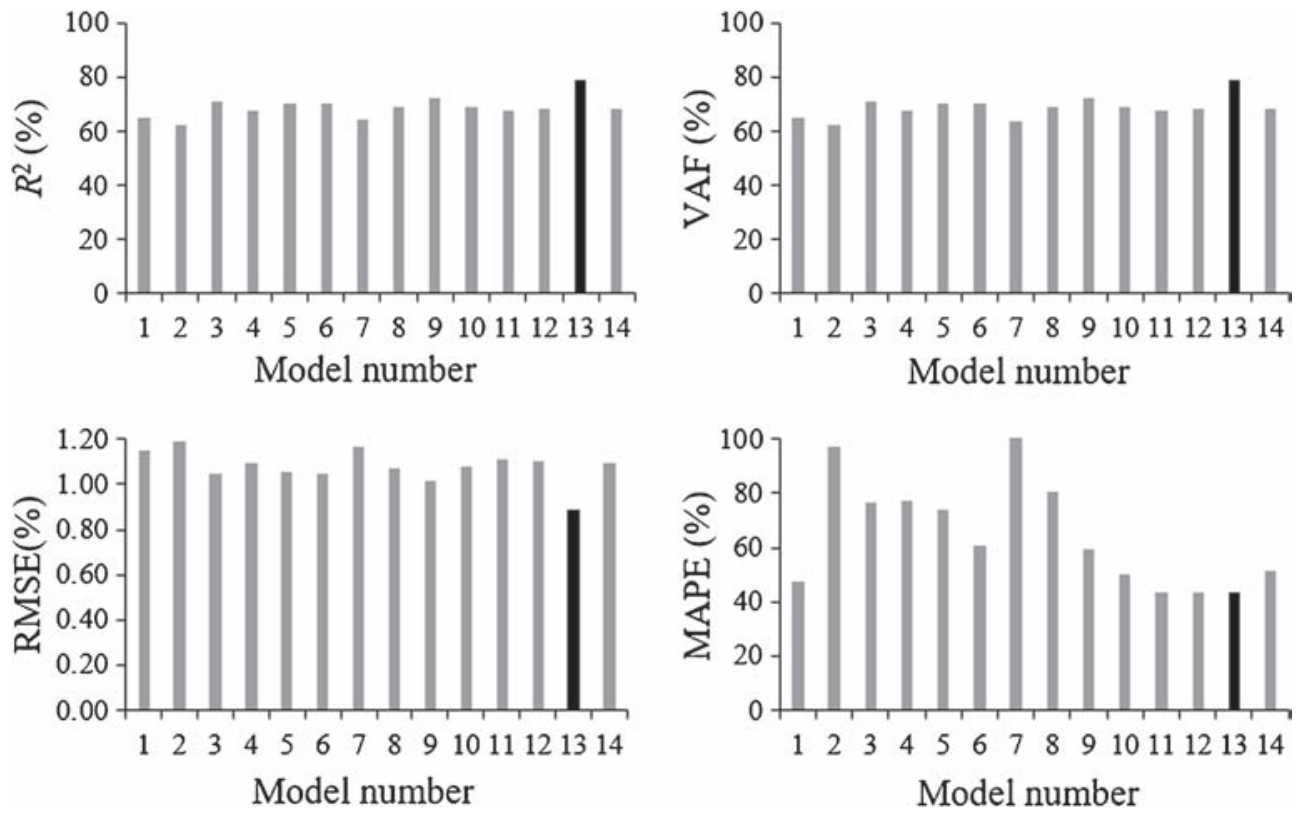

Figure 6. SARIMA-GEP model results obtained for all input combinations.
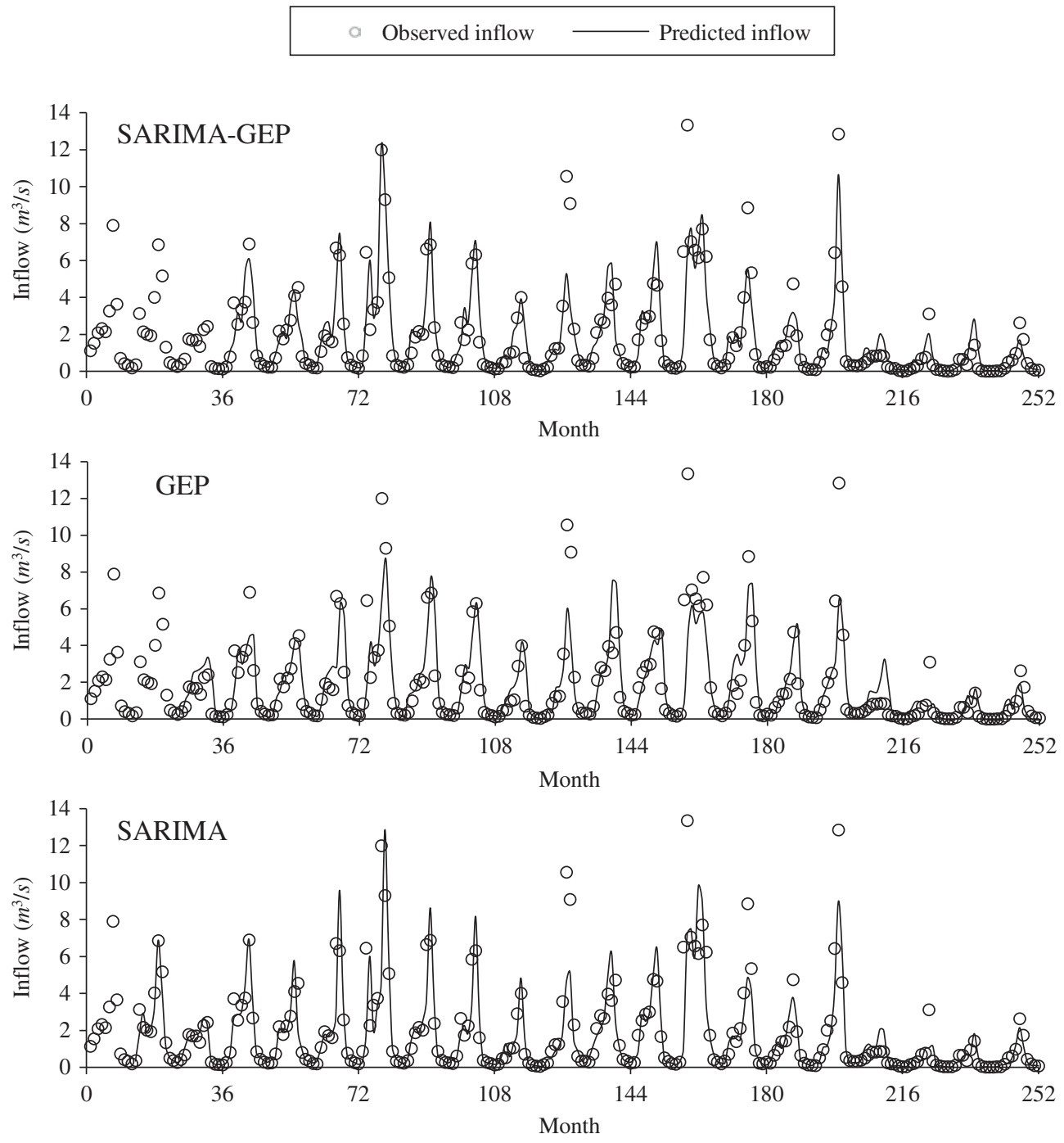

Figure 7. SARIMA-GEP, GEP and SARIMA model results obtained in the training period. 
Considering the orders of the SARIMA model parameters are between 0 and 5 , and by applying one seasonal time differencing $(\mathrm{D}=1)$ in this model, 1296 models were obtained. Then the AICc criterion was calculated for all models. The best model obtained was $\operatorname{SARIMA}(1,0,2)(0,1,1)_{12}$ with a criterion of -356.5 . Table 4 displays the t-student test significance results for the coefficients obtained from this model. Since the $P_{\tau}$ value for all coefficients is less than the $5 \%$ confidence level, the model has significant coefficients. As a result, $\operatorname{SARIMA}(1,0,2)(0,1,1)_{12}$ was selected as the best model.

In the hybrid model, the SARIMA residuals should be modelled as nonlinear components. In order to ensure the non-linearity of the residual series, the Box-Pierce test was used. The results of this test are presented in table 5 . It is clear that for each step considered, the $P_{Q^{*}}$ value is greater than the $5 \%$ confidence level. Hence, the residual series are independent in terms of linear connection.
In addition to evaluating the non-linearity of residuals, the existence of deterministic components was studied as well. Since the original series includes a strong periodic term, this term may exist in residuals accordingly. This fact was determined by plotting a cumulative periodogram (figure 4). It is observed that all the points obtained in this graph are near the exact line of points $(0,0)$ and $(1$, $0.5)$ and between the Kolmogorov-Smirnov confidence limit at the $25 \%$ confidence level. Therefore, in addition to being non-linear, the linear model residuals do not include a periodic term. Consequently, in the proposed hybrid process, the series can be modelled by nonlinear models.

By adding the removed deterministic terms to the SARIMA $\left(\hat{S}_{L}(t)\right)$ model results, the modelled linear term $\left(\hat{Q}_{L}(t)\right)$ is obtained. This is done by applying reverse differencing to $\hat{S}_{L}(t)$. The residual of this model that was considered a nonlinear component of the stochastic term $\left(S_{N}(t)\right)$ was modelled
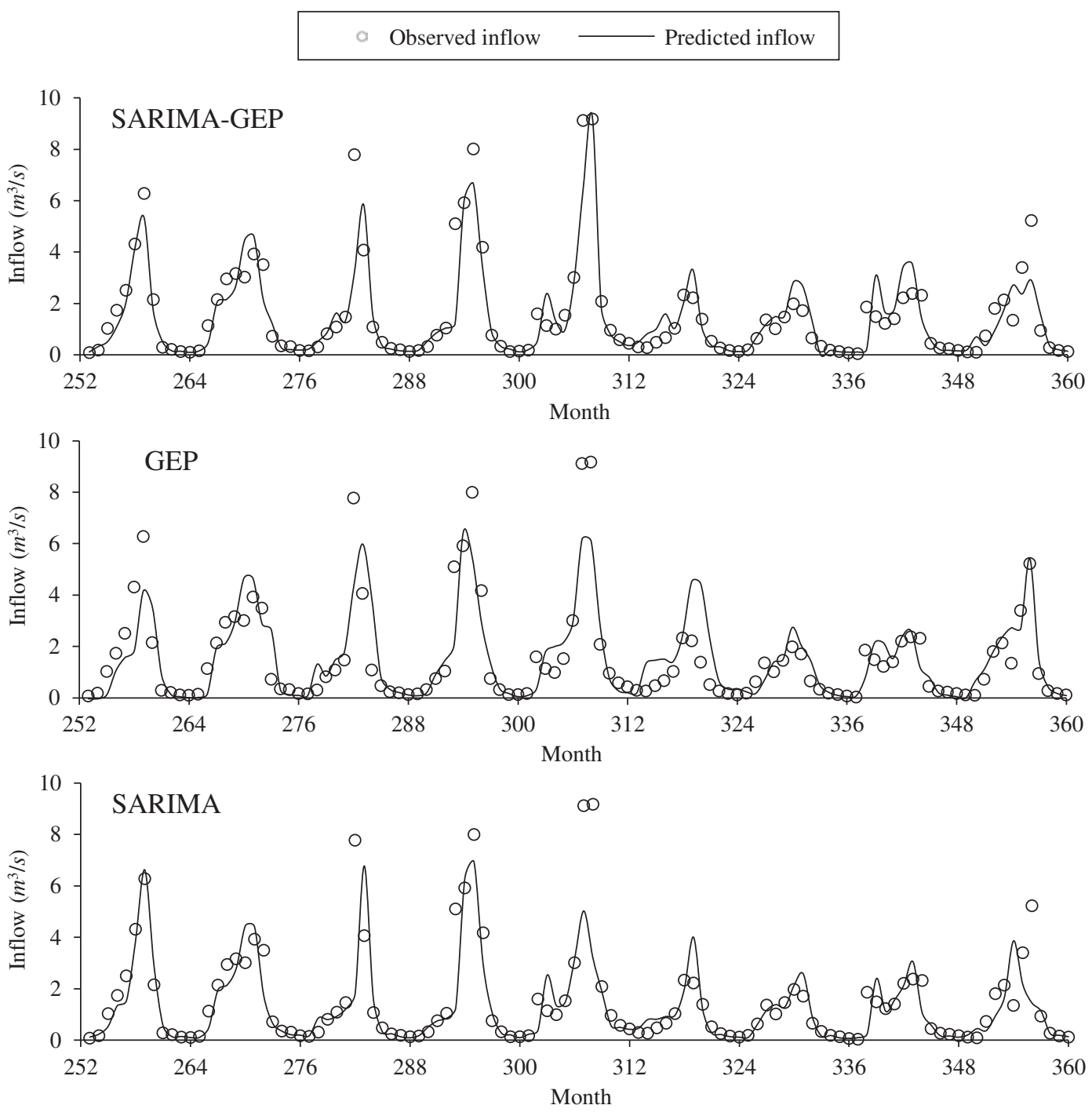

Figure 8. SARIMA-GEP, GEP and SARIMA model results obtained in the testing period. 
with GEP. Therefore, the hybrid SARIMA-GEP model was obtained. To compare the results of this model with SARIMA-ANN, $S_{N}(t)$ was also modelled with ANN. For this purpose, 14 input combinations as presented in table 1 were used for each model. With every input combination and by using the best settings of each model, the optimum result was obtained. Subsequently, the results of the 14 input combinations were compared.

\section{Results and discussion}

After modelling and predicting the monthly inflow with the SARIMA-GEP model, the results were compared with the SARIMA-ANN, GEP and ANN model results. The results obtained for each model with all 14 input combinations are shown in table 1 . Figure 5 shows the MAPE criterion values for each the 14 input combinations for all models. A comparison of the individual and hybrid model results signifies that the hybrid models were more accurate than the individual models in most cases. The best ANN model results are related to combinations 9 and 13, for SARIMA-ANN 12 and 13, for GEP 9, 13 and 14 and for SARIMA-GEP 11, 12 and 13 . It can be seen that case 13 is common for all models. It should be noted that based on the above criteria, the most accurate result for each hybrid model is related to the same input group. The lowest error values were observed for SARIMA-GEP. Therefore, a further evaluation of the impact of inputs on model results is provided in figure 6 . This figure displays the results of the evaluation criteria of each of the 14 input combinations for the SARIMA-GEP model. It is clear that all evaluation criteria for group 13 produced the best results. Therefore, this input combination can be considered the best combination in monthly inflow modelling. This group includes five inputs with a maximum delay of two years: $S_{N}(t-1), S_{N}(t-2)$, $S_{N}(t-3), S_{N}(t-12), S_{N}(t-24)$. In individual models, instead of the nonlinear term $\left(S_{N}\right)$, the directly observed discharge $(Q)$ was used.

Figures 7 and 8 show the values obtained with the SARIMA-GEP model based on the best input combination compared with the SARIMA and GEP models in training and testing (respectively). It is evident from these figures that SARIMA and GEP performed weakly in estimating peak discharge. However, with the highest increase in discharge prediction accuracy, the SARIMA-GEP model was able to improve the accuracy of each individual model.

To evaluate the performance of the proposed hybrid model, figure 9 is provided. This figure shows the prediction results of SARIMA-GEP and other models using the best input combination
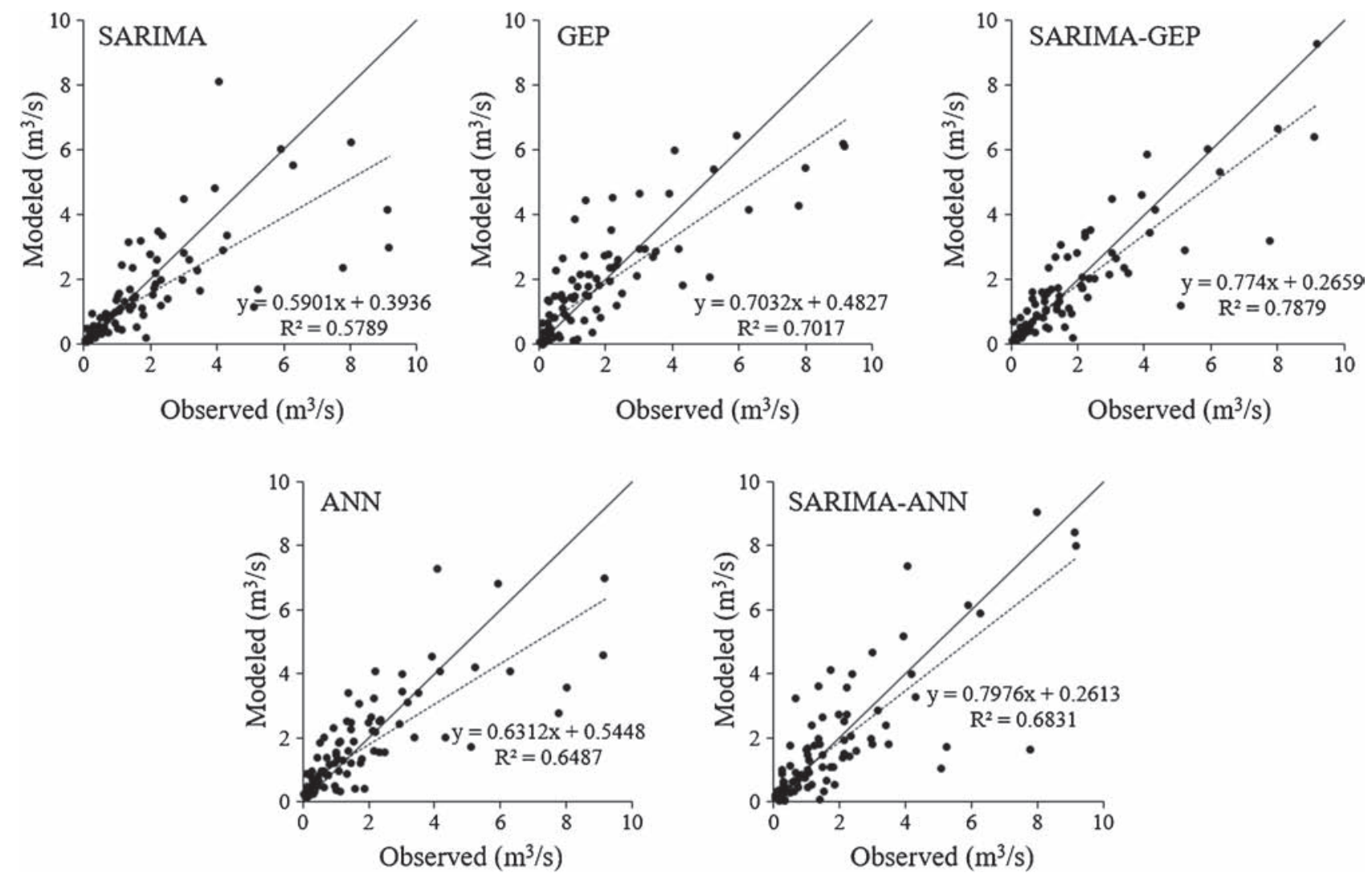

Figure 9. Prediction results of all models. 
Table 6. Evaluation criterion results for predicted inflow.

\begin{tabular}{lccccr}
\hline Model & $R^{2}$ & VAF & RMSE & MAPE & CRM \\
\hline SARIMA-GEP & 78.8 & 78.8 & 0.89 & 43.4 & 0.053 \\
GEP & 70.2 & 70.2 & 1.05 & 69.4 & -0.017 \\
SARIMA-ANN & 68.3 & 66.4 & 1.12 & 56.6 & 0.032 \\
ANN & 64.9 & 64.8 & 1.14 & 73.8 & 0.014 \\
SARIMA & 57.8 & 57.9 & 1.27 & 39.8 & 0.154 \\
\hline
\end{tabular}

(group 13). The figure indicates that the results of each combination model are better than the individual constituent models. Moreover, the individual models were weak in predicting the peak discharge but this weakness was overcome by the combination models. In this figure, it is clear that SARIMA-GEP obtained points with the highest correlation and the best-fit line.

The results obtained for the evaluation criteria for all models in the testing period are presented in table 6. It can be seen from the table that according to all criteria, the SARIMA-GEP model performed better than the individual models. In addition, SARIMA-GEP was much more accurate than the hybrid SARIMA-ANN model and is thus a good alternative. Another important result is that SARIMA-ANN was less accurate than GEP based on all criteria, except MAPE. It means that the GEP model was better than SARIMA-ANN, or at least similar. Therefore, there may be an individual model that performs more accurately than a hybrid model. Since the researchers only compared the hybrid SARIMA-ANN model with individual models, this point was hidden so far; but SARIMAANN was more accurate than either SARIMA or ANN. Thus, both hybrid models were more accurate than the individual constituent models.

It can be seen in table 6 that the SARIMA model performed worse than other models based on all criteria except MAPE. However, the MAPE value for this model was better than the hybrid SARIMA-GEP model. This indicates the higher accuracy of the linear SARIMA model in modelling discharge at low levels since the MAPE criterion is based on the relative error that is more sensitive to small numbers. This leads to the conclusion that monthly inflow on regular days of the year and for basic discharge has a linear property, and for torrential discharge, it has a nonlinear property. Hence, the nature of this hydrological phenomenon was established better.

\section{Conclusion}

In this study, a combined process consisting of four steps, namely pre-processing, linear modelling, evaluation of model non-linearity, and linear and nonlinear residual modelling was presented. With this process, two hybrid models, i.e., SARIMAGEP and SARIMA-ANN were obtained. The results of the two models were compared with individual GEP, ANN and SARIMA models. The results of all combination and individual models showed that using inputs of 1, 2, 3, 12 and 24 prior months led to the best results. Therefore, the mentioned months are the most influential on monthly inflow level prediction. The new hybrid combination SARIMAGEP showed the most accurate results among all hybrid and individual models $\left(R^{2}=78.8, \mathrm{VAF}=\right.$ $78.8, \mathrm{RMSE}=0.89, \mathrm{MAPE}=43.4, \mathrm{CRM}=0.053)$. The GEP method $\left(R^{2}=70.2, \mathrm{VAF}=70.2, \mathrm{RMSE}=\right.$ $1.05, \mathrm{MAPE}=69.4, \mathrm{CRM}=-0.017)$ produced better results than ANN and SARIMA-ANN with the best input combination (model 13). In long-term prediction, SARIMA-GEP performed much better, compared with each individual model. As basic discharge has linear features, the SARIMA model could predict this parameter very well. Applying other computational intelligence methods is suggested (e.g., adaptive neuro-fuzzy inference system, extreme learning machines) for modelling the nonlinear component of monthly inflow.

\section{Acknowledgement}

The authors would like to express their appreciation to Ms. Maya Binder for final editing of the English text.

\section{References}

Abebe A and Foerch G 2008 Stochastic simulation of the severity of hydrological drought; Water Environ. J. 22 2-10.

Aksoy H, Unal N, Eris E and Yuce M 2013 Stochastic modeling of Lake Van water level time series with jumps and multiple trends; Hydrol. Earth Syst. Sci. 17 22972303.

Alavi A H and Gandomi A H 2011 A robust data mining approach for formulation of geotechnical engineering systems; Eng. Comput. 28 242-274.

Ali S M 2013 Time series analysis of Baghdad rainfall using ARIMA method; Iraqi J. Sci. 54 1136-1142.

Box G E and Pierce D A 1970 Distribution of residual autocorrelations in autoregressive-integrated moving average time series models; J. Am. Statist. Assoc. 65 1509-1526.

Chebaane M, Salas J D and Boes D C 1995 Product periodic autoregressive processes for modeling intermittent monthly streamflows; Water. Resour. Res. 31 1513-1518.

Cryer J and Chen K 2008 Time Series Analysis with Applications in $R$; Springer Texts in Statistics, New York, USA.

Dagum E B, Lothian J R and Morry M 1975 A test of independence of the residuals based on the cumulative periodogram; Seasonal Adjustment Methods Unit, Statistics Canada.

Ebtehaj I and Bonakdari H 2013 Evaluation of sediment transport in sewer using artificial neural network; Eng. Appl. Comput. Fluid Mech. 7 382-392. 
Ebtehaj I, Bonakdari H, Zaji A H, Azimi H and Sharifi A 2015 Gene expression programming to predict the discharge coefficient in rectangular side weirs; Appl. Soft. Comput. 35 618-628.

Faruk D Ö 2010 A hybrid neural network and ARIMA model for water quality time series prediction; Eng. Appl. Artif. Intel. 23 586-594.

Ferreira C 2001 Algorithm for solving gene expression programming: A new adaptive problems; Complex Systems 13 87-129.

Ferreira C 2006 Gene Expression Programming: Mathematical modeling by an artificial intelligence; Springer Berlin Heidelberg, New York, USA.

Filho J, Affonso C M and Oliveira R C 2014 Energy price forecasting in the North Brazilian market using NNARIMA model and explanatory variables; In: Proc. Comput. Intel. Eng. Solut. (CIES), Orlando, Florida, USA, 9-12 December.

Firat M 2008 Comparison of artificial intelligence techniques for river flow forecasting; Hydrol. Earth. Syst. Sci. 12 $123-139$.

He Z, Wen X, Liu H and Du J 2014 A comparative study of artificial neural network, adaptive neuro fuzzy inference system and support vector machine for forecasting river flow in the semiarid mountain region; J. Hydrol. 509 379-386.

Hirsch R M and Slack J R 1984 A nonparametric trend test for seasonal data with serial dependence; Water Resour. Res. 20 727-732.

Kashyap R L and Rao A R 1976 Dynamic stochastic models from empirical data; Mathematics in Science and Engineering, New York, USA.

Khoshbin F, Bonakdari H, Ashraf Talesh S H, Ebtehaj I, Zaji A H and Azimi H 2016 Adaptive neuro-fuzzy inference system multi-objective optimization using the genetic algorithm/singular value decomposition method for modelling the discharge coefficient in rectangular sharp-crested side weirs; Eng. Optimiz. 48 933-948.

Kilinç I and Cigizoglu K 2005 Reservoir management using artificial neural networks; In Proc. 14th. Reg. Director. DSI (State Hydraulic Works), Istanbul, Turkey.

Kisi Ö 2004 River flow modeling using artificial neural networks; J. Hydrol. Eng. 9 60-63.

Kisi Ö and Cigizoglu H K 2007 Comparison of different ANN techniques in river flow prediction; Civil Eng. Environ. Syst. 24 211-231.

Kisi O, Dailr A H, Cimen M and Shiri J 2012a Suspended sediment modeling using genetic programming and soft computing techniques; J. Hydrol. 450 48-58.

Kisi O, Shiri J and Nikoofar B 2012b Forecasting daily lake levels using artificial intelligence approaches; Comput. Geosci. 41 169-180.

Kothari M and Gharde K 2015 Application of ANN and fuzzy logic algorithms for streamflow modelling of Savitri catchment; J. Earth Syst. Sci. 124 933-943.

Koutroumanidis T, Ioannou K and Arabatzis G 2009 Predicting fuelwood prices in Greece with the use of ARIMA models, artificial neural networks and a hybrid ARIMA-ANN model; Energ. Policy 37 3627-3634.

Koza J R 1992 Genetic programming: On the programming of computers by means of natural selection; MIT press, London, England.

Levenberg K 1944 A method for the solution of certain nonlinear problems in least squares; Quart. Appl. Math. 2(2) $164-168$.
Liu H, H-q Tian and Li Y-f 2012 Comparison of two new ARIMA-ANN and ARIMA-Kalman hybrid methods for wind speed prediction; Appl. Energ. 98 415424.

Lohani A, Kumar R and Singh R 2012 Hydrological time series modeling: A comparison between adaptive neurofuzzy, neural network and autoregressive techniques; $J$. Hydrol. 442 23-35.

Makridakis S, Wheelwright S C and Hyndman R J 2008 Forecasting methods and applications; John Wiley \& Sons, Hoboken, New Jersey, USA.

Mann H B and Whitney D R 1947 On a test of whether one of two random variables is stochastically larger than the other; Ann. Math. Stat., pp. 50-60.

Marco J B, Harboe R and Salas J D 2012 Stochastic hydrology and its use in water resources systems simulation and optimization; Springer Science \& Business Media, B V, Peniscola, Spain.

Moeeni H and Bonakdari H 2016 Forecasting monthly inflow with extreme seasonal variation using the hybrid SARIMA-ANN model; Stoch. Env. Res. Risk. A, doi: 10.1007/s00477-016-1273-z.

Mohammadi K, Eslami H and Dardashti S D 2005 Comparison of regression, ARIMA and ANN models for reservoir inflow forecasting using snowmelt equivalent (a case study of Karaj); J. Agric. Sci. Technol. 7 17-30.

Mohan S and Vedula S 1995 Multiplicative seasonal ARIMA model for longterm forecasting of inflows; Water Resour. Manag. 9 115-126.

Nourani V, Kisi Ö and Komasi M 2011 Two hybrid artificial intelligence approaches for modeling rainfall-runoff process; J. Hydrol. 402 41-59.

Salas J, Delleur J, Yevjevich V and Lane W 1988 Applied Modeling of Hydrologic Time Series; Water Resources Publications, Colorado, USA.

Sharma N, Zakaullah M, Tiwari H and Kumar D 2015 Runoff and sediment yield modeling using ANN and support vector machines: A case study from Nepal watershed; Model Earth Syst. Environ. 1 1-8.

Valipour M 2015 Long-term runoff study using SARIMA and ARIMA models in the United States; Meteorol. Appl. 22 592-598.

Valipour M, Banihabib M E and Behbahani S M R 2013 Comparison of the ARMA, ARIMA, and the autoregressive artificial neural network models in forecasting the monthly inflow of Dez dam reservoir; J. Hydrol. 476433 441.

Wang W, Van Gelder P and Vrijling J 2005 Long-memory in streamflow processes of the Yellow river; In: Proc. IWA Int. Conf. Water Economics Statistics and Finance Rethymno, Greece, 8-10 July, 2005.

Wang W-C, Chau K-W, Cheng C-T and Qiu L 2009 A comparison of performance of several artificial intelligence methods for forecasting monthly discharge time series; J. Hydrol. 374 294-306.

Yurekli K and Kurunc A 2005 Performances of stochastic approaches in generating low streamflow data for drought analysis; J. Spat. Hydrol. 5.

Yurekli K, Kurunc A and Ozturk F 2005 Application of linear stochastic models to monthly flow data of Kelkit Stream; Ecol. Model. 183 67-75.

Zhang G P 2003 Time series forecasting using a hybrid ARIMA and neural network model; Neurocomputing $\mathbf{5 0}$ 159-175. 